\title{
MAGIC UPPER LIMITS ON THE VERY HIGH ENERGY EMISSION FROM GAMMA-RAY BURSTS
}

J. Albert ${ }^{1}$ E. Aliu ${ }^{2}$ H. Anderhub,${ }^{3}$ P. Antoranz,${ }^{4}$ A. Armada,${ }^{2}$ C. Baixeras, ${ }^{5}$ J. A. Barrio, ${ }^{4}$ H. Bartko, ${ }^{6}$ D. Bastieri ${ }^{7}$ J. Becker, ${ }^{8}$ W. Bednarek, ${ }^{9}$ K. Berger, ${ }^{1}$ C. Bigongiari, ${ }^{7}$ A. Biland, ${ }^{3}$ R. K. Bock, ${ }^{6,7}$ P. Bordas, ${ }^{10}$ V. Bosch-Ramon, ${ }^{10}$ T. Bretz, ${ }^{1}$ I. Britvitch, ${ }^{3}$ M. Camara, ${ }^{4}$ E. Carmona, ${ }^{6}$ A. Chilingarian, ${ }^{11}$ S. Ciprini, ${ }^{12}$ J. A. Coarasa, ${ }^{6}$ S. Commichau, ${ }^{3}$ J. L. Contreras, ${ }^{4}$ J. Cortina, ${ }^{2}$ M. T. Costado, ${ }^{13}$ V. Curtef, ${ }^{8}$ V. Danielyan, ${ }^{11}$ F. Dazzi, ${ }^{7}$ A. De Angelis, ${ }^{14}$ C. Delgado, ${ }^{13}$ R. De los Reyes, ${ }^{4}$ B. De Lotto, ${ }^{14}$ E. Domingo-Santamaría, ${ }^{2}$ D. Dorner, ${ }^{1}$ M. Doro, ${ }^{7}$ M. Errando, ${ }^{2}$ M. Fagiolini, ${ }^{15}$ D. Ferenc, ${ }^{16}$ E. Fernández, ${ }^{2}$ R. Firpo, ${ }^{2}$ J. Flix, ${ }^{2}$ M. V. Fonseca, ${ }^{4}$ L. Font,${ }^{5}$ M. Fuchs, ${ }^{6}$ N. Galante, ${ }^{6}$ R. García-López, ${ }^{13}$ M. Garczarczyk, ${ }^{6}$ M. Gaug, ${ }^{7}$ M. Giller, ${ }^{9}$ F. Goebel, ${ }^{6}$ D. Hakobyan, ${ }^{11}$ M. Hayashida, ${ }^{6}$ T. Hengstebeck, ${ }^{17}$ A. Herrero, ${ }^{13}$ D. Höhne, ${ }^{1}$ J. Hose, ${ }^{6}$ C. C. Hsu, ${ }^{6}$ P. Jacon, ${ }^{9}$ T. Jogler, ${ }^{6}$ O. Kalekin, ${ }^{17}$ R. Kosyra, ${ }^{6}$ D. Kranich, ${ }^{3}$ R. Kritzer, ${ }^{1}$ A. Laille, ${ }^{16}$ T. Lenisa, ${ }^{14}$ P. Liebing, ${ }^{6}$ E. Lindfors, ${ }^{12}$ S. Lombardi, ${ }^{7}$ F. Longo, ${ }^{14}$ J. López, ${ }^{2}$ M. López, ${ }^{4}$ E. Lorenz, ${ }^{3,6}$ P. Majumdar, ${ }^{6}$ G. Maneva, ${ }^{18}$ K. Mannheim, ${ }^{1}$ O. Mansutti, ${ }^{14}$ M. Mariotti, ${ }^{7}$ M. Martínez, ${ }^{2}$ D. Mazin, ${ }^{6}$ C. Merck, ${ }^{6}$ M. Meucci, ${ }^{15}$ M. Meyer, ${ }^{1}$ J. M. Miranda, ${ }^{4}$ R. Mirzoyan, ${ }^{6}$ S. Mizobuchi, ${ }^{6}$ A. Moralejo, ${ }^{2}$ K. Nilsson, ${ }^{12}$ J. Ninkovic, ${ }^{6}$ E. Oña-Wilhelmi, ${ }^{2}$ N. Otte,${ }^{6}$ I. Oya, ${ }^{4}$ D. Paneque, ${ }^{6}$ M. Panniello, ${ }^{13}$ R. Paoletti, ${ }^{15}$ J. M. Paredes, ${ }^{10}$ M. Pasanen, ${ }^{12}$ D. Pascoli, ${ }^{7}$ F. Pauss,${ }^{3}$ R. Pegna, ${ }^{15}$ M. Persic, ${ }^{14,19}$ L. Peruzzo, ${ }^{7}$ A. Piccioli, ${ }^{15}$ M. Poller, ${ }^{1}$ E. Prandini, ${ }^{7}$ N. Puchades, ${ }^{2}$ A. Raymers, ${ }^{11}$ W. Rhode, ${ }^{8}$ M. Ribó, ${ }^{10}$ J. Rico, ${ }^{2}$ M. Rissi, ${ }^{3}$ A. Robert, ${ }^{5}$ S. Rügamer, ${ }^{1}$ A. Saggion, ${ }^{7}$ A. Sánchez,${ }^{5}$ P. SArtori, ${ }^{7}$ V. Scalzotto, ${ }^{7}$ V. Scapin, ${ }^{7}$ R. Schmitt, ${ }^{1}$ T. Schweizer, ${ }^{6}$ M. Shayduk, ${ }^{6,17}$ K. Shinozaki, ${ }^{6}$ S. N. Shore, ${ }^{20}$ N. Sidro, ${ }^{2}$ A. Sillanpää, ${ }^{12}$ D. Sobczynska, ${ }^{9}$ A. Stamerra, ${ }^{15}$ L. S. Stark, ${ }^{3}$ L. Takalo, ${ }^{12}$ P. Temnikov, ${ }^{18}$ D. Tescaro, ${ }^{2}$ M. Teshima, ${ }^{6}$ N. Tonello, ${ }^{6}$ D. F. Torres, ${ }^{2,21}$ N. Turini, ${ }^{15}$ H. Vankov, ${ }^{18}$ V. Vitale, ${ }^{14}$ R. M. Wagner, ${ }^{6}$ T. Wibig, ${ }^{9}$ W. Wittek, ${ }^{6}$ R. ZANin, ${ }^{2}$ AND J. ZAPATERO ${ }^{5}$ Received 2006 December 14; accepted 2007 May 22

\begin{abstract}
During its first data cycle, between 2005 and the beginning of 2006, the fast repositioning system of the MAGIC telescope allowed the observation of nine different gamma-ray bursts as possible sources of very high energy $\gamma$-rays. These observations were triggered by alerts from Swift, HETE-2, and INTEGRAL; they started as quickly as possible after the alerts and lasted for several minutes, with an energy threshold varying between 80 and $200 \mathrm{GeV}$, depending on the zenith angle of the burst. No evidence for gamma signals was found, and upper limits for the flux were derived for all events using the standard analysis chain of MAGIC. For the bursts with measured redshifts, the upper limits are compatible with a power-law extrapolation, when the intrinsic fluxes are evaluated taking into account the attenuation due to the scattering in the metagalactic radiation field.
\end{abstract}

Subject headings: gamma rays: bursts — gamma rays: observations

Online material: color figures

\section{INTRODUCTION}

The physical origin of the enigmatic gamma-ray bursts (GRBs) is still under debate, 40 years after their discovery (see Mészáros 2006 for a recent review). The possible detection of radiation in the very high energy (VHE) region (extending between a few tens of $\mathrm{GeV}$ and a few tens of $\mathrm{TeV}$ ) will lead to a deeper understanding of the acceleration mechanisms and the emission processes from GRBs. The $\gamma$-ray emission observed by the Energetic Gamma Ray Experiment Telescope (EGRET) in some case extends up to the VHE band (Hurley et al. 1994; Dingus 1995; González et al.

\footnotetext{
1 Universität Würzburg, D-97074 Würzburg, Germany.

2 Institut de Física d'Altes Energies, Edifici Cn., E-08193 Bellaterra (Barcelona), Spain.

${ }^{3}$ ETH Zurich, CH-8093 Zurich, Switzerland.

4 Universidad Complutense, E-28040 Madrid, Spain.

5 Universitat Autònoma de Barcelona, E-08193 Bellaterra, Spain.

6 Max-Planck-Institut für Physik, D-80805 München, Germany.

7 Università di Padova and INFN, I-35131 Padova, Italy.

8 Universität Dortmund, D-44227 Dortmund, Germany.

9 University of Łódź, PL-90236 Lodz, Poland.

10 Universitat de Barcelona, E-08028 Barcelona, Spain.

11 Yerevan Physics Institute, AM-375036 Yerevan, Armenia.
}

2003), favoring the hypothesis of a highly relativistic source of nonthermal radiation situated in an optically thin region (Piran 1999); more insight, however, can be gained by a clear signal detection in the VHE region, or the evaluation of stringent upper limit in this energy band.

Several observations of GRBs at energies above $100 \mathrm{GeV}$ have been attempted (Götting et al. 2003; Zhou et al. 2003), without showing any indication of a signal. This is due to relatively low sensitivity, as in satellite-borne detectors, or to high energy thresholds, as in the previous generation of Cerenkov telescopes or in particle detector arrays. Only a few tentative detections of radiation above $0.1 \mathrm{TeV}$ were reported by MILAGRITO for GRB

\footnotetext{
12 Tuorla Observatory, Turku University, FI-21500 Piikkiö, Finland.

13 Instituto de Astrofisica de Canarias, E-38200 La Laguna, Tenerife, Spain.

14 Università di Udine, and INFN Trieste, I-33100 Udine, Italy.

15 Università di Siena, and INFN Pisa, I-53100 Siena, Italy.

16 University of California, Davis, CA 95616-8677.

17 Humboldt-Universität zu Berlin, D-12489 Berlin, Germany.

18 Institute for Nuclear Research and Nuclear Energy, BG-1784 Sofia, Bulgaria.

19 INAF/Osservatorio Astronomico and INFN Trieste, I-34131 Trieste, Italy.

20 Università di Pisa, and INFN Pisa, I-56126 Pisa, Italy.

21 ICREA and Institut de Cienciès de l'Espai, IEEC-CSIC, E-08193 Bellaterra,
} Spain. 
970417a (Atkins et al. 2000), or by the GRAND array for GRB 971110 (Poirer et al. 2003).

Upper limits on the prompt or delayed emission of GRBs were also set by Whipple (Connaughton et al. 1997; Horan et al. 2007), MILAGRO (Atkins et al. 2005; Saz Parkinson et al. 2006a, 2006b, 2007), STACEE (Jarvis et al. 2005), and HEGRA AIROBICC (Padilla et al. 1998).

Imaging atmospheric Cerenkov telescopes (IACTs) of the latest generation achieve a better flux sensitivity and a lower energy threshold, and thus are better suited to detect VHE $\gamma$-rays; on the other hand, their small fields of view permit unguided observations only by virtue of serendipitous detection, and they have to rely on an external trigger, such as that provided by automated satellite link to the GRB Coordinates Network (GCN), which broadcasts the coordinates of events triggered and selected by dedicated satellite detectors.

Among the new Cerenkov telescopes, MAGIC (Mirzoyan 2005) is best suited for the detection of the prompt emission of GRBs, because of its low energy threshold, its large effective area, and in particular, its capability for fast slewing (Bretz et al. 2003). The low trigger threshold, currently $50 \mathrm{GeV}$ at zenith, should allow the observation of GRBs even at large redshift, as lower energy radiation can effectively reach Earth without interacting much with the metagalactic radiation field (MRF). Moreover, in its fastslewing mode, MAGIC can be repositioned within $30 \mathrm{~s}$ to any position on the sky; in case of a target-of-opportunity alert by GCN, an automated procedure takes only few seconds to terminate any pending observation, validate the incoming signal, and start slewing toward the GRB position. Until now, the maximal repositioning time has been $\sim 100 \mathrm{~s}$. In two cases, this allowed the placement of upper limits on the GRB flux even during prompt emission (Galante et al. 2005; Albert et al. 2006; Morris et al. 2007).

The detection of VHE radiation from GRBs is important for comparing different theoretical models. Emission in the $\mathrm{GeV}-\mathrm{TeV}$ range in the prompt and delayed phases is predicted by several authors (see Zhang \& Mészáros 2001; Pe'er \& Waxman 2004; Razzaque et al. 2004 for a detailed analysis). Possible processes comprise leptonic and hadronic models: inverse Compton (IC) scattering by electrons in internal (Papathanassoiu \& Mészáros 1996; Pilla \& Loeb 1998) or external shocks (Mészáros et al. 1994), IC in the afterglow shocks (Dermer et al. 2000; Zhang \& Mészáros 2001; Derishev et al. 2001; Wang et al. 2001), IC by electrons responsible for optical flashes (Beloborodov 2005), pure electron-synchrotron (Zhang \& Mészáros 2001) and protonsynchrotron emission ( Totani 2000), photon-pion production (Waxman 1995; Böttcher \& Dermer 1998; Chiang \& Dermer 1999; Li et al. 2002; Fragile et al. 2004), and neutron cascades (Bahcall \& Mészáros 2000; Derishev et al. 1999; Rossi et al. 2006).

During the early afterglow phase, the recent observations by the Swift satellite of X-ray flares lasting $10^{3}-10^{5} \mathrm{~s}$ (Burrows et al. 2005 ) suggested an extended activity in the central engine of the GRB, and thus emission from late internal shocks (Kobayashi et al. 2007) or from refreshed shocks due to energy injections at later times (Guetta et al. 2007). In some cases, the energy release of these flares can be of the same order of magnitude as the energy release in the prompt phase, as reported for GRB $050502 \mathrm{~b}$. The possibility of correlated $\gamma$-ray emission extending into the $\mathrm{GeV}-\mathrm{TeV}$ region is predicted as well, where the corresponding VHE flares are predicted to originate from IC-scattered photons in the forward shock (Wang et al. 2006). Thus, observation of the delayed activity is of particular interest, being in most cases not constrained by the alerting and slewing time, and being still connected to the investigation of the central engine dynamics.
Measurements in this energy range can be used to test all these competing models. However, as most of the observed GRBs occur at large redshift, strong attenuation of the VHE $\gamma$-ray flux is expected, as a result of the interaction with low-energy photons of the MRF (Nikishov 1961; de Jager \& Stecker 2002). The knowledge of the redshift, therefore, is important for a precise interpretation (Mannheim et al. 1996).

In this article, we report on the analysis of data collected on several GRBs followed by MAGIC during their prompt-emission and early afterglow phases.

\section{GAMMA-RAY ANALYSIS WITH THE MAGIC TELESCOPE}

The Major Atmospheric Gamma Imaging Cherenkov (MAGIC) telescope (Mirzoyan 2005), located on the Canary Island of La Palma (2200 $\mathrm{m}$ above sea level, $28^{\circ} 45^{\prime}$ north, $17^{\circ} 54^{\prime}$ west), completed its commissioning phase in early fall 2004. MAGIC is currently the largest IACT, with a $17 \mathrm{~m}$ diameter tessellated reflector dish consisting of $9640.5 \times 0.5 \mathrm{~m}^{2}$ diamond-milled aluminum mirrors. In its current configuration, the MAGIC photomultiplier camera has a trigger region of $2.0^{\circ}$ diameter (Cortina et al. 2005) and a trigger collection area for $\gamma$-rays of the order of $10^{5} \mathrm{~m}^{2}$, which increases further with the zenith angle of observation. Presently, the accessible trigger energy range spans from 50-60 GeV (at small zenith angles) to tens of TeV. The MAGIC telescope is focused to $10 \mathrm{~km}$ distance, the most likely height at which a $50 \mathrm{GeV} \gamma$-ray shower has its maximum. The accuracy in reconstructing the direction of incoming $\gamma$-rays, the point-spread function (PSF), is about $0.1^{\circ}$, slightly depending on the analysis.

The reconstructed signals are calibrated (Gaug et al. 2005) and then cleaned of spurious backgrounds from the light of the night sky using two different image-cleaning procedures: one algorithm requiring signal exceeding fixed reference levels, and a second algorithm employing additionally the reconstructed information of the arrival time (Gaug 2006). Nonphysical background images are eliminated (e.g., car flashes having triggered the readout). Events are processed by means of the MAGIC standard analysis software (Bretz et al. 2005), using the standard Hillas analysis (Hillas 1985; Fegan et al. 1997). Gamma/hadron separation is performed by means of the random forest (RF) method (Breiman 2001), a classification method that combines several parameters describing the shape of the image into a new parameter called "hadronness" (Hengstebeck 2006), the final $\gamma /$ hadron discriminator in our analysis. Monte Carlo samples are used to optimize, as a function of energy, the cuts in hadronness. The energy of the $\gamma$-ray is also estimated using an RF approach, yielding a resolution of $\sim 30 \%$ at $200 \mathrm{GeV}$. The parameter ALPHA of the Hillas analysis, which is related to the direction of the incoming shower, is not included in the calculation of hadronness, as it is used separately to evaluate the significance of a signal. If the telescope is directed at a point-like $\gamma$-ray source, as a GRB is expected to be, the ALPHA distribution of collected photons should peak at $0^{\circ}$, while it is uniform for isotropic background showers.

\section{BLIND TEST WITH CRAB NEBULA}

On 2005 October 11 at 02:17:37 UT, the INTEGRAL satellite announced GRB $051011^{22}$ at the J2000.0 position R.A. = $5^{\mathrm{h}} 34^{\mathrm{m}} 47^{\mathrm{s}}$, decl. $=+21^{\circ} 54^{\prime} 39^{\prime \prime}$. A few hours later, INTEGRAL sent a new GCN (Mereghetti \& Mowlavi 2005) stating that GRB 051011 was in fact the Crab Nebula. Thus, in a blind test, we acquired $2814 \mathrm{~s}$ of events coming from the Crab Nebula, the standard

22 IBAS alert 2673; see http://ibas.iasf-milano.inaf.it 
TABLE 1

Summary of GRBs Observed by MAGIC from 2005 April to 2006 March

\begin{tabular}{|c|c|c|c|c|c|c|c|c|}
\hline Number & Burst & Satellite & $\begin{array}{c}T_{0} \\
\text { (UT) }\end{array}$ & $\begin{array}{l}\Delta T_{\text {alert }} \\
\text { (s) }\end{array}$ & $\begin{array}{l}\Delta T_{\text {start }} \\
\text { (s) }\end{array}$ & $\begin{array}{l}t_{\text {slewing }} \\
\text { (s) }\end{array}$ & $\begin{array}{c}\text { Data } \\
\text { (minutes) }\end{array}$ & $\begin{array}{l}\text { Zenith Angle } \\
\text { (deg) }\end{array}$ \\
\hline$\ldots \ldots \ldots \ldots \ldots$ & GRB 050421 & Swift & $04: 11: 52$ & 58 & 108 & 26 & 75 & $\sim 52$ \\
\hline 2 & GRB $050502 a$ & INTEGRAL & $02: 13: 57$ & 39 & 689 & 223 & 87 & $\sim 30$ \\
\hline 3 & GRB 050505 & Swift & $23: 22: 21$ & 540 & 717 & 90 & 101 & $\sim 49$ \\
\hline 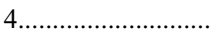 & GRB 050509a & Swift & $01: 46: 29$ & 16 & 131 & 108 & 119 & $\sim 58$ \\
\hline 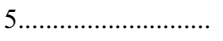 & GRB 050713a & Swift & 04:29:02 & 13 & 40 & 17 & 37 & $\sim 49$ \\
\hline 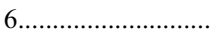 & GRB 050904 & Swift & $01: 51: 44$ & 82 & 145 & 54 & 147 & $\sim 24$ \\
\hline 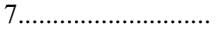 & GRB 060121 & HETE-2 & $22: 24: 54$ & 15 & 583 & $\ldots$ & 53 & $\sim 48$ \\
\hline 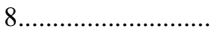 & GRB 060203 & Swift & $23: 55: 35$ & 171 & 268 & 84 & 43 & $\sim 44$ \\
\hline 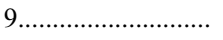 & GRB 060206 & Swift & $04: 46: 53$ & 16 & 59 & 35 & 49 & $\sim 13$ \\
\hline
\end{tabular}

Notes.- Here $\Delta T_{\text {alert }}$ stands here for the time delay after $T_{0}$ until the burst coordinates were received from the GCN; $\Delta T_{\text {start }}$ is the total time delay before the observation could be started, of which $t_{\text {slewing }}$ is the time lost for repositioning the telescope. Data column shows the total amount of data taken.

source of $\gamma$-rays at VHE energies. The analysis yielded a $14 \sigma$ signal above $350 \mathrm{GeV}$ (Scapin et al. 2007), showing that MAGIC can observe, at $5 \sigma$ level, spectra of $5 \mathrm{crab}(1 \mathrm{crab}=6.57 \times$ $10^{-10} \mathrm{~cm}^{-2} \mathrm{~s}^{-1}$ above $100 \mathrm{GeV}$ ) of intensity in $40 \mathrm{~s}$, if above $300 \mathrm{GeV}$, and in $90 \mathrm{~s}$ if below $300 \mathrm{GeV}$.

\section{GRBs OBSERVED BY MAGIC DURING ITS FIRST OBSERVATION CYCLE}

An automatic alert system has been operational from 2004 July 15 . Since then, about 200 GRBs were detected by HETE-2, INTEGRAL, and Swift, out of which about 100 contained GRB coordinates. Time delays to the onset of the burst were of the order of several seconds to tens of minutes. During the first MAGIC data cycle, between 2005 April and 2006 March, nine GRBs were observed by MAGIC during the prompt or the early afterglow emission phase, as listed in Table 1. In two cases the prompt alerting by the GCN and the fast reaction of the MAGIC telescope allowed to take data not only on the early afterglow, but also on part of the prompt emission of the burst. These two bursts are GRB 0507013a and GRB 050904, and will be considered separately.

\subsection{The Properties of Observed GRBs}

Table 2 summarizes the properties of observed GRBs by MAGIC according to the information distributed through the GCN Circular service.

GRB 050421 was detected by the Burst Alert Telescope (BAT) on board Swift (Barbier et al. 2005a). The other telescope on board Swift, the X-Ray Telescope (XRT), observed the burst in the 0.2-
$10 \mathrm{keV}$ range from $T_{0}+97 \mathrm{~s}$ and detected two X-ray flares at $T_{0}+110$ and $T_{0}+154 \mathrm{~s}$ (Godet et al. 2006). Figure 1 shows the X-ray light curve of this burst. It can be seen that the MAGIC observation window is overlapped with the XRT ones on the X-ray afterglow. In particular, the two small X-ray flares are in the observation window of MAGIC. No optical counterpart was observed; thus GRB 050421 has been cataloged as a dark burst.

GRB 050502a was triggered by INTEGRAL; no X-ray counterpart was observed, but an optical afterglow followed the burst (Gotz 2005a, 2005b; Durig 2005). GRB 050505 was triggered by BAT and its light curve presented three short spikes at $T_{0}+$ 23.3, $T_{0}+30.4$, and $T_{0}+50.4 \mathrm{~s}$ (Hurkett et al. 2005a; Hullinger et al. 2005). Both X-ray and optical observations followed the burst, but there was no simultaneous observation by MAGIC and the other instruments on board the satellite, as shown in Figure 2.

GRB 050509a was triggered by BAT and later XRT detected an X-ray counterpart (Hurkett et al. 2005b; Barbier et al. 2005b). GRB 060121 is the only short burst observed by MAGIC and was triggered by HETE-2 (Arimoto et al. 2006; Golenetskii et al. 2006). XRT observed the afterglow as well and detected a fading X-ray source inside the HETE-2 error box (Mangano et al. 2006), as shown in Figure 3. Unfortunately, there is no overlap between MAGIC observation and XRT observation. An optical counterpart was not confirmed by TNG, which did detect, however, a weak source inside the XRT error box (Malesani et al. 2006). Moreover, HST gave no evidence of an optical afterglow, although the burst lay close to a faint red galaxy at high redshift (Levan et al. 2006).

TABLE 2

Main Properties of GRBs Observed by MAGiC

\begin{tabular}{|c|c|c|c|c|c|c|}
\hline Number & Burst & Trigger Number & Energy Range & $\begin{array}{l}T_{90} \\
(\mathrm{~s})\end{array}$ & $\begin{array}{l}\text { Fluence } \\
\left(\mathrm{erg} \mathrm{cm}^{-2} \text { ) }\right.\end{array}$ & $z$ \\
\hline 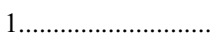 & GRB 050421 & 115135 & $15-350 \mathrm{keV}$ & 10 & $1.8 \times 10^{-7}$ & .. \\
\hline $2 \ldots \ldots \ldots \ldots \ldots \ldots \ldots$ & GRB $050502 a$ & 2484 & $20-200 \mathrm{keV}$ & 20 & $1.4 \times 10^{-6}$ & 3.79 \\
\hline 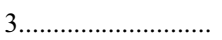 & GRB 050505 & 117504 & $15-350 \mathrm{keV}$ & 60 & $4.1 \times 10^{-6}$ & 4.27 \\
\hline ....................... & GRB 050509a & 118707 & $15-350 \mathrm{keV}$ & 13 & $4.6 \times 10^{-7}$ & \\
\hline 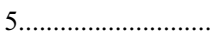 & GRB 050713a & 145675 & $15-350 \mathrm{keV}$ & 70 & $9.1 \times 10^{-6}$ & \\
\hline 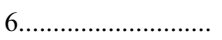 & GRB 050904 & 153514 & $15-350 \mathrm{keV}$ & 225 & $5.4 \times 10^{-6}$ & 6.29 \\
\hline 7 & GRB 060121 & 4010 & $0.02-1 \mathrm{MeV}$ & 2 & $4.7 \times 10^{-6}$ & $\cdots$ \\
\hline 8 & GRB 060203 & 180151 & $15-350 \mathrm{keV}$ & 60 & $8.5 \times 10^{-7}$ & $\ldots$ \\
\hline 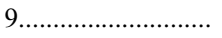 & GRB 060206 & 180455 & $15-350 \mathrm{keV}$ & 11 & $8.4 \times 10^{-7}$ & 4.05 \\
\hline
\end{tabular}

Notes.-The fourth column shows the typical energy range of the detector on board the satellite, while the fifth, sixth, and seventh columns show the corresponding measured duration $T_{90}$, fluence, and redshift. 


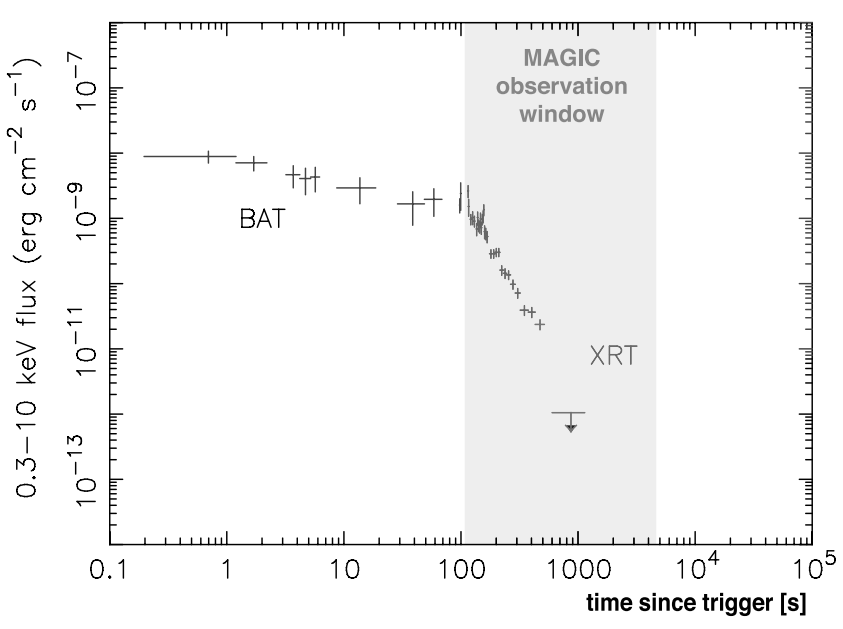

FIG. 1.-Flux of GRB 050421 measured by BAT and XRT. The shaded area represents the MAGIC observation time window and the overlap with Swift data. [See the electronic edition of the Journal for a color version of this figure.]

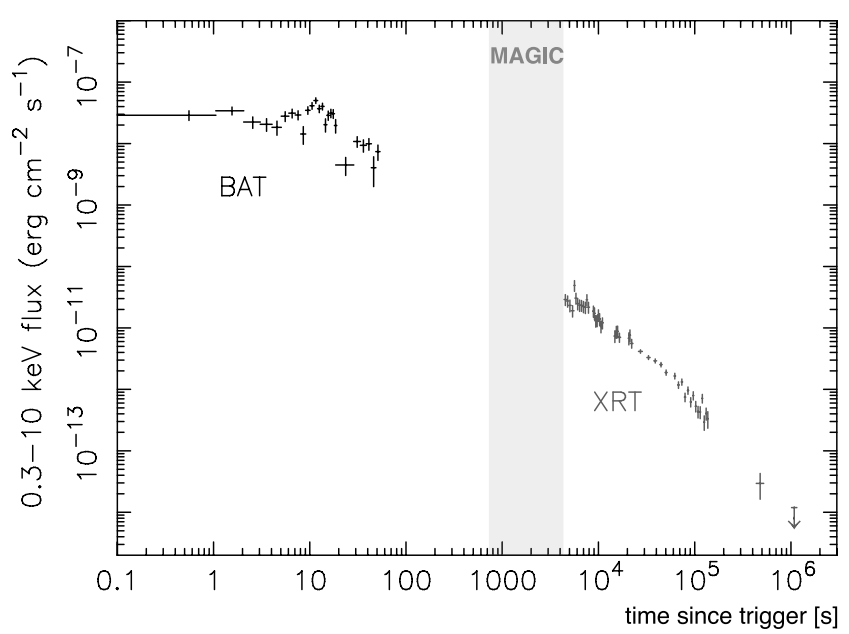

FIG. 2.-Flux of GRB 050505 measured by BAT and XRT. The shaded area represents the MAGIC observation time window, starting $717 \mathrm{~s}$ after the burst onset. [See the electronic edition of the Journal for a color version of this figure.]

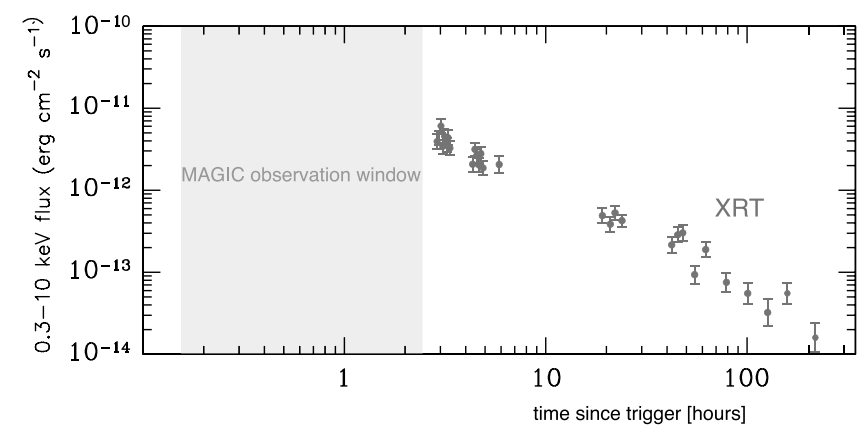

Fig. 3.-Flux of the afterglow of GRB 060121 measured by the Swift XRT detector. The MAGIC observation window is shown as shaded area. [See the electronic edition of the Journal for a color version of this figure.]

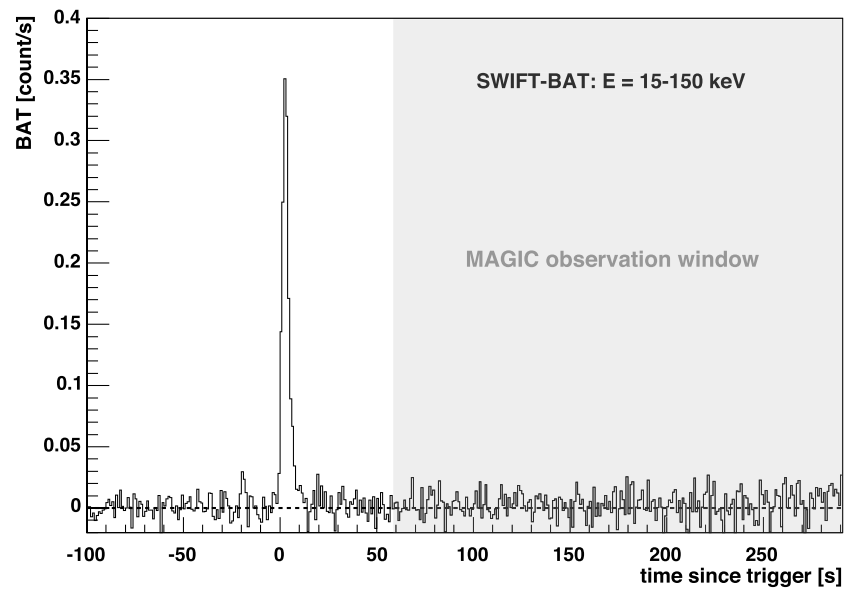

FIG. 4.- - Light curve of GRB 060206 measured by BAT, sampled in bins of $1 \mathrm{~s}$. The beginning of MAGIC observation is shown in the shaded area. [See the electronic edition of the Journal for a color version of this figure.]

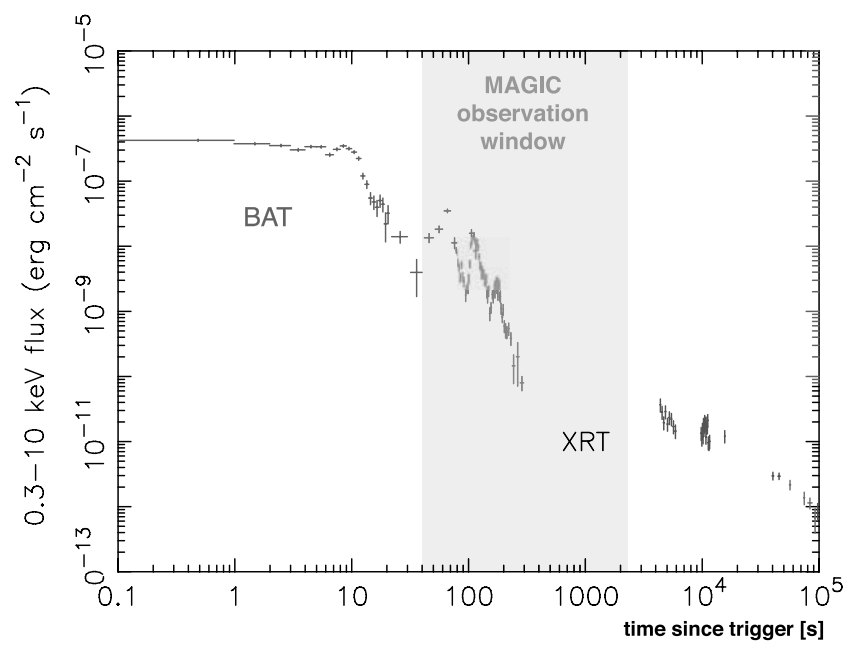

FIG. 5.-Flux of the prompt and afterglow emission of GRB 050713a measured by BAT and XRT. The shaded area illustrates the observation time window of MAGIC. [See the electronic edition of the Journal for a color version of this figure.]

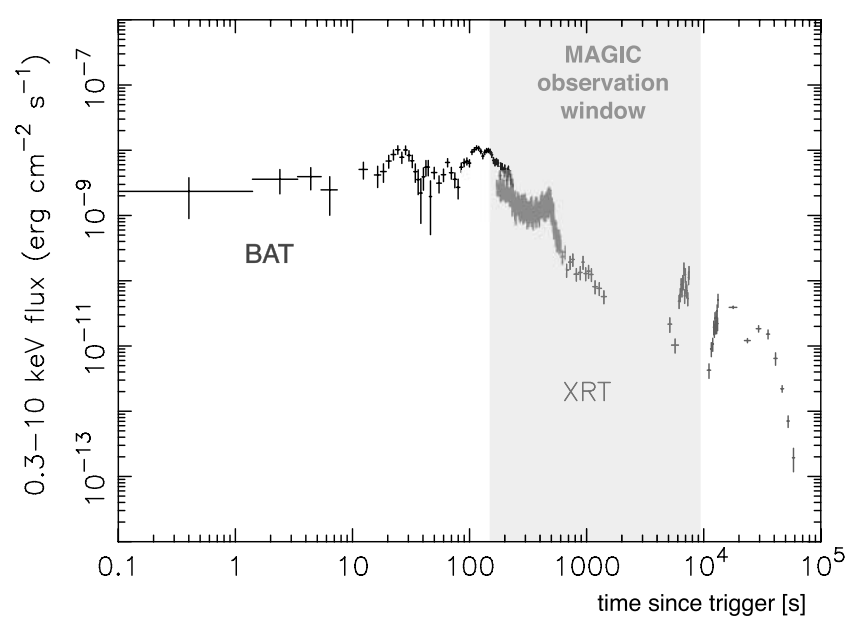

FIG. 6.-Flux measured by BAT and XRT in the prompt and afterglow emission of GRB 050904. The MAGIC observation window is shown in the shaded area. [See the electronic edition of the Journal for a color version of this figure.] 

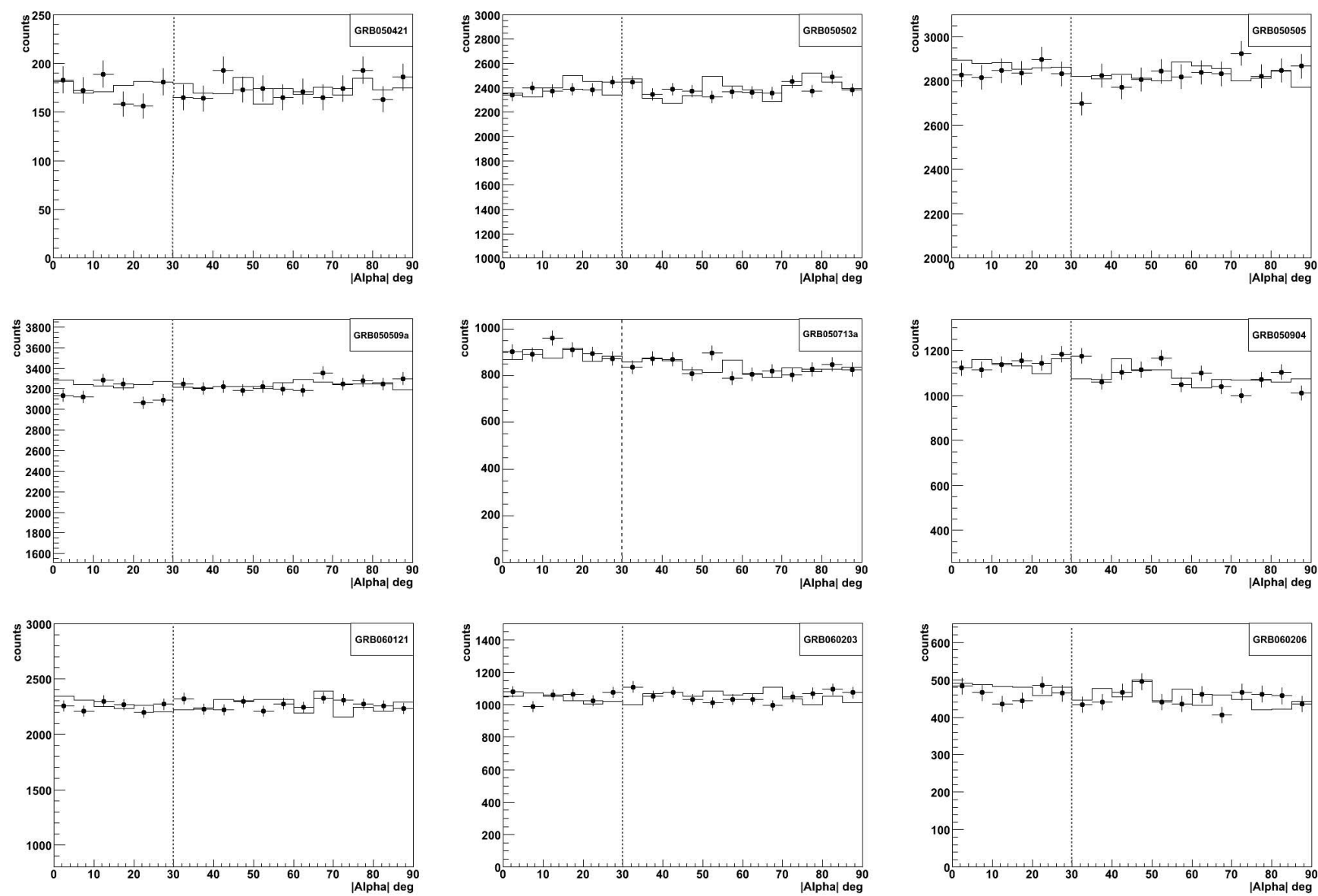

FIG. 7. - ALPHA plots of all nine GRBs for the complete data set of each burst. Points with error bars refer to the burst data; the line refers to the background. [See the electronic edition of the Journal for a color version of this figure.]

The last two bursts, GRB 060203 and GRB 060206, are both long bursts triggered by BAT (Barthelmy et al. 2006; Morris et al. 2006). MAGIC data overlap with XRT data on the X-ray afterglow of GRB 060206, immediately after the BAT data, as shown in Figure 4. No evidence of flares or of the jet break have been claimed, but optical observations provided a high redshift value (Palmer et al. 2006; Aoki et al. 2006).

\subsection{GRB 050713a and GRB 050904 Prompt-Emission Observations}

GRB 050713a is of particular interest, being the first burst observed by MAGIC during its prompt emission (Albert et al. 2006). On 2005 July 13 at 04:29:02 UT, the BAT instrument detected a burst located at R.A. $=21^{\mathrm{h}} 22^{\mathrm{m}} 09.53^{\mathrm{s}}$, decl. $=+77^{\circ} 04^{\prime} 29.50^{\prime \prime} \pm 3^{\prime}$ (Falcone et al. 2005). The MAGIC alert system received and validated the alert $13 \mathrm{~s}$ after the burst; data taking started $40 \mathrm{~s}$ after the burst original time $T_{0}$ (Galante et al. 2005). The burst was classified as a bright burst by Swift with a duration of $T_{90}=70 \pm 10 \mathrm{~s}$. The brightest part of the keV emission occurred within $T_{0}+20 \mathrm{~s}$, three smaller peaks followed at $T_{0}+50, T_{0}+65$, and $T_{0}+$ $105 \mathrm{~s}$, while a preburst peak took place at $T_{0}-60 \mathrm{~s}$ (see Fig. 5). The spectrum, over the interval from $T_{0}-70$ to $T_{0}+121 \mathrm{~s}$, can be fitted with a power law with photon index $-1.58 \pm 0.07$ and yields a fluence of $9.1 \times 10^{-6} \mathrm{erg} \mathrm{cm}^{-2}$ in the $15-350 \mathrm{keV}$ range (Palmer et al. 2005). The burst triggered also the Konus-Wind satellite (Golenetskii et al. 2005), which measured the spectrum of the burst during the first $16 \mathrm{~s}$, which is the duration of the first big peak as reported by Swift. In the local coordinate system of
MAGIC, GRB 050713a was located at an azimuth angle of $-6^{\circ}$ (near north) and a zenith angle of $50^{\circ}$. The sky region of the burst was observed during 37 minutes, until twilight.

GRB 050904 is also of particular interest, being the second and the latest burst with prompt emission observed by MAGIC. It was triggered at 01:51:44 UT by BAT; the coordinates were R.A. $=0{ }^{\mathrm{h}} 54^{\mathrm{m}} 50.79^{\mathrm{s}}$, decl. $=+14^{\circ} 05^{\prime} 09.42^{\prime \prime} \pm 3^{\prime}$ (Cummings et al. 2005). XRT slewed promptly and started the observation at $T_{0}+161 \mathrm{~s}$, revealing an uncataloged fading source. It is a long burst $\left(T_{90}=225 \mathrm{~s}\right)$, with a total fluence of $5.4 \times 10^{-6} \mathrm{erg} \mathrm{cm}^{-2}$ in the 15-150 keV range (Sakamoto et al. 2005). This burst is the most distant burst ever observed, with an estimated redshift $z=$ 6.29 (Kawai et al. 2005). Its X-ray light curve (see Fig. 6) shows a clear X-ray flare at $T_{0}+466 \mathrm{~s}$ (Mineo et al. 2005), and is thus in the MAGIC observation window.

\section{RESULTS}

All nine GRBs were analyzed using the MAGIC standard analysis described above. In this work the image-cleaning algorithm using also arrival time information was used, as it is more robust. For each GRB a dedicated OFF-source data set was selected on the basis of being compatible with the $\mathrm{ON}$ data with respect to several parameters: zenith angle, as the effective area depends strongly on it; local brightness of the sky, depending mostly on Moon phase and zenith angle; and trigger rate, depending mostly on atmospheric transparency and on hardware settings. Loose preliminary cuts were used to remove unphysical events. After training of the RF, for each burst a "hadronness" cut was 

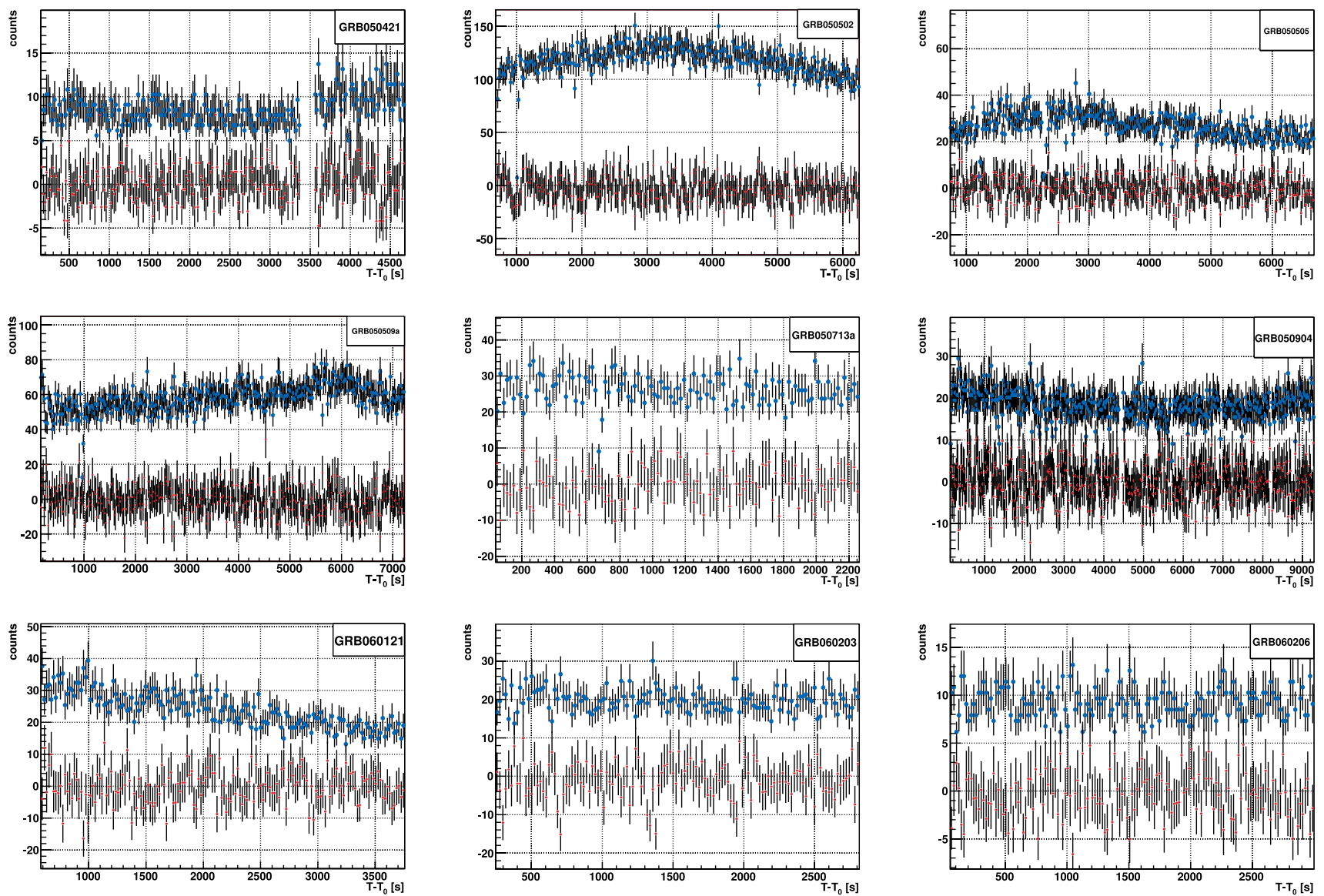

FIG. 8. - Light curves of all nine GRBs for the complete data set of each burst. The background rate of GRB 050502 is particularly high because of a higher night-sky background due to the Moon light.

applied that granted about $90 \%$ efficiency on $\gamma$-ray events according to the corresponding Monte Carlo, in order to keep high statistics of possible $\gamma$-ray events.

The analysis showed no evident signal excess, as can be seen in Figure 7. For each burst the ALPHA plot over the whole data set and for reconstructed energies greater than $100 \mathrm{GeV}$ is shown. The ALPHA distributions of the GRB data sets are flat, as expected from background hadronic events, and are compatible with

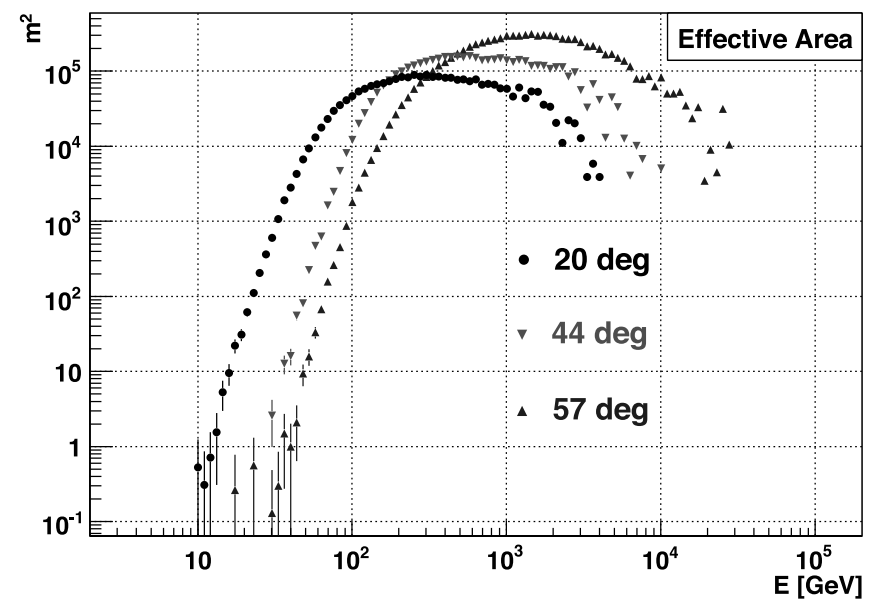

FIG. 9.-Effective area of MAGIC, after typical cuts used in this analysis, for three different zenith angles. [See the electronic edition of the Journal for a color version of this figure.] the corresponding OFF-source data set. No excess in the signal region, i.e., for ALPHA $<30^{\circ}$, can be seen.

Nor was any excess seen using a temporal analysis: the entire data-taking interval was divided into $20 \mathrm{~s} \mathrm{time} \mathrm{bins} \mathrm{and} \mathrm{the} \mathrm{num-}$ ber of potential $\gamma$-ray events was extracted from the ALPHA distribution; they are shown in the light curves of Figure 8, where red filled circles denote the excess events, and blue open circles the background events (offset by 5 from excess counts in order to make the plot more readable). The distributions of excess events remain zero on average during the observation, and no significant variation of the sample average is visible with time.

Upper limits have been derived for the first 30 minutes of each burst using the Rolke approach (Rolke et al. 2005) in six reconstructed energy bins: $80-120 \mathrm{GeV}, 120-175 \mathrm{GeV}, 175-$ $225 \mathrm{GeV}, 225-300 \mathrm{GeV}, 300-400 \mathrm{GeV}$, and $400-1000 \mathrm{GeV}$. A systematic uncertainty of $30 \%$ on the efficiency has been considered in the upper limit calculation. In every reconstructed energy bin the upper limit in number of excess, calculated with the Rolke approach, has been converted into flux units using the effective area, as explained in the Appendix. The typical effective area of MAGIC for different zenith angles is shown in Figure 9. Table 3 summarizes the upper limits derived for all nine GRBs during the first 30 minutes of data taking.

\section{DISCUSSION}

A preliminary estimate of the observability of GRBs by the MAGIC telescope had originally been derived using the fourth BATSE catalog (Bastieri et al. 2005; Galante 2006). The GRB 
TABLE 3

Derived Fluence Upper Limits for the First 30 Minutes of Data of Nine Gamma-Ray Bursts

\begin{tabular}{|c|c|c|c|c|}
\hline \multirow{2}{*}{$\begin{array}{c}\text { ENERGY BIN } \\
(\mathrm{GeV})\end{array}$} & \multirow{2}{*}{$\begin{array}{c}\text { ENERGY } \\
(\mathrm{GeV})\end{array}$} & \multicolumn{3}{|c|}{ FLUENCE UPPER Limit } \\
\hline & & $\mathrm{cm}^{-2} \mathrm{keV}^{-1}$ & $\operatorname{erg~} \mathrm{cm}^{-2}$ & crab \\
\hline \multicolumn{5}{|l|}{ GRB 050421: } \\
\hline $175-225 \ldots$ & 212.5 & $5.26 \times 10^{-16}$ & $3.80 \times 10^{-8}$ & 0.20 \\
\hline $225-300 \ldots \ldots$ & 275.8 & $3.64 \times 10^{-16}$ & $4.43 \times 10^{-8}$ & 0.27 \\
\hline $300-400 \ldots \ldots \ldots \ldots \ldots$ & 366.4 & $5.21 \times 10^{-17}$ & $1.12 \times 10^{-8}$ & 0.08 \\
\hline $400-1000 \ldots \ldots \ldots \ldots \ldots$ & 658.7 & $2.07 \times 10^{-17}$ & $1.41 \times 10^{-8}$ & 0.14 \\
\hline \multicolumn{5}{|l|}{ GRB 050502: } \\
\hline $120-175 \ldots \ldots \ldots \ldots \ldots$ & 152.3 & $1.67 \times 10^{-15}$ & $6.21 \times 10^{-8}$ & 0.27 \\
\hline $175-225 \ldots \ldots \ldots \ldots \ldots$ & 219.3 & $2.83 \times 10^{-15}$ & $2.18 \times 10^{-7}$ & 1.15 \\
\hline $225-300 \ldots \ldots \ldots \ldots \ldots$ & 275.8 & $1.13 \times 10^{-15}$ & $1.37 \times 10^{-7}$ & 0.83 \\
\hline $300-400 \ldots \ldots \ldots \ldots \ldots$ & 360.8 & $7.57 \times 10^{-17}$ & $1.58 \times 10^{-8}$ & 0.11 \\
\hline $400-1000 \ldots$ & 629.1 & $5.62 \times 10^{-17}$ & $3.56 \times 10^{-8}$ & 0.35 \\
\hline \multicolumn{5}{|l|}{ GRB 050505: } \\
\hline $175-225 \ldots$ & 212.9 & $2.03 \times 10^{-15}$ & $1.48 \times 10^{-7}$ & 0.76 \\
\hline $225-300 \ldots \ldots$ & 275.1 & $2.66 \times 10^{-15}$ & $3.22 \times 10^{-7}$ & 1.94 \\
\hline $300-400 \ldots \ldots \ldots \ldots \ldots \ldots$ & 363.6 & $5.28 \times 10^{-16}$ & $1.11 \times 10^{-7}$ & 0.79 \\
\hline $400-1000 \ldots$. & 704.1 & $1.85 \times 10^{-17}$ & $1.46 \times 10^{-8}$ & 0.15 \\
\hline \multicolumn{5}{|l|}{ GRB 050509a: } \\
\hline $175-225 \ldots \ldots$ & 215.1 & $1.04 \times 10^{-15}$ & $7.69 \times 10^{-8}$ & 0.40 \\
\hline $225-300 \ldots \ldots$ & 273.4 & $1.39 \times 10^{-15}$ & $1.67 \times 10^{-7}$ & 1.00 \\
\hline $300-400 \ldots \ldots$. & 362.8 & $7.74 \times 10^{-16}$ & $1.63 \times 10^{-7}$ & 1.15 \\
\hline $400-1000 \ldots \ldots \ldots \ldots \ldots$ & 668.5 & $1.69 \times 10^{-16}$ & $1.21 \times 10^{-7}$ & 1.22 \\
\hline \multicolumn{5}{|l|}{ GRB 050713a: } \\
\hline $120-175 \ldots \ldots$. & 169.9 & $3.63 \times 10^{-15}$ & $1.68 \times 10^{-7}$ & 0.76 \\
\hline $175-225 \ldots \ldots \ldots \ldots \ldots$ & 212.5 & $1.12 \times 10^{-15}$ & $8.08 \times 10^{-8}$ & 0.42 \\
\hline $225-300 \ldots \ldots$. & 275.8 & $2.07 \times 10^{-15}$ & $2.52 \times 10^{-7}$ & 1.52 \\
\hline $300-400 \ldots \ldots \ldots \ldots \ldots \ldots$ & 366.4 & $3.33 \times 10^{-16}$ & $7.16 \times 10^{-8}$ & 0.51 \\
\hline $400-1000 \ldots \ldots \ldots \ldots \ldots$ & 658.7 & $2.24 \times 10^{-17}$ & $1.55 \times 10^{-8}$ & 0.15 \\
\hline \multicolumn{5}{|l|}{ GRB 050904: } \\
\hline $80-120 \ldots \ldots$ & 85.5 & $9.06 \times 10^{-15}$ & $1.06 \times 10^{-7}$ & 0.32 \\
\hline $120-175 \ldots \ldots \ldots \ldots \ldots$ & 140.1 & $3.00 \times 10^{-15}$ & $9.42 \times 10^{-8}$ & 0.38 \\
\hline $175-225 \ldots \ldots \ldots \ldots \ldots \ldots$ & 209.9 & $2.18 \times 10^{-15}$ & $1.53 \times 10^{-7}$ & 0.79 \\
\hline $225-300 \ldots \ldots \ldots \ldots \ldots$ & 268.9 & $5.82 \times 10^{-16}$ & $6.74 \times 10^{-8}$ & 0.40 \\
\hline $300-400 \ldots \ldots \ldots \ldots \ldots \ldots$ & 355.2 & $5.01 \times 10^{-16}$ & $1.11 \times 10^{-7}$ & 0.71 \\
\hline $400-1000 \ldots \ldots$. & 614.9 & $1.26 \times 10^{-16}$ & $7.63 \times 10^{-8}$ & 0.73 \\
\hline \multicolumn{5}{|l|}{ GRB 060121: } \\
\hline $120-175 \ldots \ldots$ & 151.3 & $2.64 \times 10^{-15}$ & $9.67 \times 10^{-8}$ & 0.41 \\
\hline $175-225 \ldots \ldots \ldots \ldots \ldots$ & 212.8 & $6.57 \times 10^{-16}$ & $4.76 \times 10^{-8}$ & 0.25 \\
\hline $225-300 \ldots \ldots$ & 273.7 & $2.13 \times 10^{-16}$ & $2.56 \times 10^{-8}$ & 0.15 \\
\hline $300-400 \ldots \ldots \ldots \ldots \ldots \ldots$ & 367.7 & $4.47 \times 10^{-16}$ & $9.66 \times 10^{-8}$ & 0.69 \\
\hline $400-1000 \ldots \ldots \ldots \ldots \ldots$ & 636.4 & $4.84 \times 10^{-17}$ & $3.14 \times 10^{-8}$ & 0.31 \\
\hline \multicolumn{5}{|l|}{ GRB 060203: } \\
\hline $120-175 \ldots \ldots \ldots \ldots \ldots \ldots$ & 151.5 & $1.10 \times 10^{-14}$ & $4.03 \times 10^{-7}$ & 1.71 \\
\hline $175-225 \ldots \ldots \ldots \ldots \ldots$ & 219.5 & $5.07 \times 10^{-16}$ & $3.91 \times 10^{-8}$ & 0.21 \\
\hline $225-300 \ldots \ldots \ldots \ldots \ldots$ & 274.0 & $1.57 \times 10^{-16}$ & $1.88 \times 10^{-8}$ & 0.11 \\
\hline $300-400 \ldots \ldots \ldots \ldots \ldots \ldots$ & 365.3 & $3.54 \times 10^{-16}$ & $7.56 \times 10^{-8}$ & 0.54 \\
\hline $400-1000 \ldots \ldots \ldots \ldots \ldots$ & 639.5 & $4.45 \times 10^{-17}$ & $2.91 \times 10^{-8}$ & 0.29 \\
\hline \multicolumn{5}{|l|}{ GRB 060206: } \\
\hline $80-120 \ldots \ldots \ldots \ldots \ldots \ldots$ & 85.5 & $1.23 \times 10^{-14}$ & $1.44 \times 10^{-7}$ & 0.44 \\
\hline $120-175 \ldots \ldots \ldots \ldots \ldots$ & 139.9 & $9.83 \times 10^{-16}$ & $3.08 \times 10^{-8}$ & 0.13 \\
\hline $175-225 \ldots \ldots \ldots \ldots \ldots \ldots$ & 210.3 & $5.50 \times 10^{-16}$ & $3.89 \times 10^{-8}$ & 0.20 \\
\hline $225-300 \ldots \ldots \ldots \ldots \ldots \ldots$ & 269.2 & $3.65 \times 10^{-16}$ & $4.23 \times 10^{-8}$ & 0.25 \\
\hline $300-400 \ldots \ldots \ldots \ldots \ldots$ & 355.4 & $6.47 \times 10^{-16}$ & $1.31 \times 10^{-7}$ & 0.91 \\
\hline $400-1000 \ldots \ldots \ldots \ldots \ldots$ & 614.0 & $2.88 \times 10^{-17}$ & $1.74 \times 10^{-8}$ & 0.17 \\
\hline
\end{tabular}

Notes.-The first column shows the reconstructed energy bins in which the analysis has been done. The second column shows the true energy at which the upper limits have been calculated, and is the energy giving the average flux upper limit in the reconstructed energy bin. The last column shows the upper limit value in crab units. 
spectra were extended to GeV energies with a simple power law and using the observed high-energy spectral index. The extrapolated fluxes were finally compared to the estimated MAGIC sensitivity. Setting conservative cuts on observation times and significances, and assuming an energy threshold of $30 \mathrm{GeV}$, a $5 \sigma$ signal rate of 0.5-2 per year was obtained for an assumed observation delay between 15 and $60 \mathrm{~s}$ and a BATSE trigger rate of $\sim 360$ per year. Taking into account the rate of GRBs (Guetta et al. 2005) and extrapolating GRB spectra, as observed by BATSE, to VHE using an unbroken power law of reasonable power index (Preece et al. 2000), it was foreseen that MAGIC could detect about one GRB per year at a $5 \sigma$ level. A maximal redshift up to $z=2$ was considered.

This estimate must be revised: the Swift alert rate is about a factor 2 lower than predicted, and it includes bursts even fainter than those observed by BATSE; also, for these bursts the effective MAGIC energy threshold at analysis level was higher than the assumed one $(\sim 80 \mathrm{GeV})$; most important, the distribution of bursts detected by Swift has a much higher median redshift than expected. As a result, the number of GRBs that MAGIC can detect is now estimated to lie in the range of $0.2-0.7$ per year. This number can be expected to increase again with the launch of GLAST and AGILE, and the increased number of alerts due to GRB monitoring by GLAST, AGILE, and Swift altogether.

\section{CONCLUSIONS}

MAGIC was able to observe part of the prompt and the early afterglow emission phase of many GRBs as a response to the alert system provided by several satellites. No excess events above $\sim 100 \mathrm{GeV}$ were detected, neither during the prompt-emission phase nor during the early afterglow. We have derived upper limits for the $\gamma$-ray flux between 85 and $1000 \mathrm{GeV}$. These limits are compatible with a naive extension of the power-law spectrum, when the redshift is known, up to hundreds of GeV.

For the first time an atmospheric Cerenkov telescope was able to perform direct rapid observations of the prompt-emission phase of GRBs. This is particularly relevant in the so-called Swift era. Although strong absorption of the high-energy $\gamma$-ray flux by the MRF is expected at high redshifts, given its sensitivity to low fluxes and its fast-slewing capabilities, the MAGIC telescope is currently expected to detect about 0.5 GRBs per year, if the GRB spectra extend to the energy domain of hundreds of $\mathrm{GeV}$, following a power law with reasonable indices.

The construction of the MAGIC telescope was mainly made possible by the support of the German BMBF and MPG, the Italian INFN, and the Spanish CICYT, to whom goes our grateful acknowledgment. We would also like to thank the IAC for the excellent working conditions at the Observatorio del Roque de los Muchachos in La Palma. This work was further supported by ETH Research Grant TH 34/04 3 and Polish MNiI Grant 1P03D01028.

Facilities: MAGIC

\section{APPENDIX}

\section{UPPER LIMIT CALCULATION}

The recorded number of events in a particular reconstructed energy bin $\Delta E_{\text {rec }}$ is

$$
N_{\Delta E_{\mathrm{rec}}}=\int_{0}^{\infty} \phi(E) A_{\mathrm{eff}}\left(E \mid \Delta E_{\mathrm{rec}}\right) d E \Delta T
$$

where $\phi(E)$ is the flux (photons $\left.\mathrm{cm}^{-2} \mathrm{~s}^{-1} \mathrm{GeV}^{-1}\right), A_{\mathrm{eff}}\left(E \mid \Delta E_{\mathrm{rec}}\right.$ ) is the effective area after all cuts, included the reconstructed energy cut $\Delta E_{\mathrm{rec}}$, and $\Delta T$ the total time interval of observation. It should be noted that the flux $\phi$ and the effective area $A$ depend on the true energy, while the cuts for the selection of the excess events $N_{\Delta E_{\mathrm{rec}}}$ and of the effective area $A_{\mathrm{eff}}\left(E \mid \Delta E_{\mathrm{rec}}\right)$ depend on the reconstructed (estimated) energy. The integral is computed in true energy $d E$. GRB:

Being the effective area $A_{\text {eff }}\left(E \mid \Delta E_{\text {rec }}\right)$ depending on energy, we must assume a spectral shape, in our case the typical power law of a

$$
\phi(E)=k \times\left(\frac{E}{E_{0}}\right)^{\beta},
$$

where $k$ is a normalization factor (photons $\mathrm{cm}^{-2} \mathrm{~s}^{-1} \mathrm{GeV}^{-1}$ ), $\beta$ is the average high-energy power-law index $\beta=-2.5$, and $E_{0}$ the pivot energy (e.g., $1 \mathrm{GeV}$ ). From equation (A1) we obtain the normalization factor as

$$
k_{*}=\frac{N_{\Delta E_{\mathrm{rec}}}}{\int_{0}^{\infty} A_{\mathrm{eff}}\left(E \mid \Delta E_{\mathrm{rec}}\right)\left(E / E_{0}\right)^{\beta} d E \Delta T} .
$$

In our case $N_{\Delta E_{\text {rec }}}$ is the upper limit in number of excess events calculated with Rolke statistics. The flux upper limit is

$$
\phi_{\mathrm{UL}}(E)=k_{*} \times\left(\frac{E}{E_{0}}\right)^{\beta} .
$$

The value of the energy $E$ is chosen in a very simple way. We calculate the energy that gives the average flux in the observed reconstructed energy bin:

$$
\langle\phi\rangle_{A_{\mathrm{eff}}}=\frac{\int_{0}^{\infty} \phi(E) A_{\mathrm{eff}}\left(E \mid \Delta E_{\mathrm{rec}}\right) d E}{\int_{0}^{\infty} A_{\mathrm{eff}}\left(E \mid \Delta E_{\mathrm{rec}}\right) d E}=\frac{N_{\Delta E_{\mathrm{rec}}}}{\int_{0}^{\infty} A_{\mathrm{eff}}\left(E \mid \Delta E_{\mathrm{rec}}\right) d E \Delta T} .
$$


From equations (A1) and (A3) we can write

$$
\langle\phi\rangle_{A_{\mathrm{eff}}}=\frac{N_{\Delta E_{\mathrm{rec}}}}{\int_{0}^{\infty} A_{\mathrm{eff}}\left(E \mid \Delta E_{\mathrm{rec}}\right)\left(E / E_{0}\right)^{\beta} d E} \times \frac{\int_{0}^{\infty} A_{\mathrm{eff}}\left(E \mid \Delta E_{\mathrm{rec}}\right)\left(E / E_{0}\right)^{\beta} d E}{\int_{0}^{\infty} A_{\mathrm{eff}}\left(E \mid \Delta E_{\mathrm{rec}}\right) d E \Delta T}=k_{*}\left\langle\left(E / E_{0}\right)^{\beta}\right\rangle_{A_{\mathrm{eff}}} .
$$

Defining $\left(E_{*} / E_{0}\right) \equiv\left\langle\left(E / E_{0}\right)^{\beta}\right\rangle_{A_{\mathrm{eff}}}^{1 / \beta}$, from equation (A4) we can calculate the average flux upper limit in the reconstructed energy bin:

$$
\phi_{\mathrm{UL}}\left(E_{*}\right)=k_{*} \times\left(\frac{E_{*}}{E_{0}}\right)^{\beta}=\left\langle\phi_{\mathrm{UL}}\right\rangle_{A_{\mathrm{eff}}} .
$$

Equation (A7) has been used to calculate the upper limits shown in Table 3.

\section{REFERENCES}

Albert, J., et al. 2006, ApJ, 641, L9

Aoki, K., et al. 2006, GCN Circ. 4703, http://gcn.gsfc.nasa.gov/gcn/gcn3/4703.gcn3

Arimoto, M., et al. 2006, GCN Circ. 4550, http:/gcn.gsfc.nasa.gov/gcn/gcn3/ 4550.gcn3

Atkins, R., et al. 2000, ApJ, 533, L119

2005, ApJ, 630, 996

Bahcall, J. N., \& Mészáros, P. 2000, Phys. Rev. Lett., 85, 1362

Barbier, L. M., et al. 2005a, GCN Circ. 3296, http://gen.gsfc.nasa.gov/gen/ gen $3 / 3296$.gen 3

- 2005b, GCN Circ. 3407, http://gcn.gsfc.nasa.gov/gen/gen3/3407.gcn3

Barthelmy, S., et al. 2006, GCN Circ. 4656, http://gen.gsfc.nasa.gov/gen/gen3/ 4656.gcn3

Bastieri, D., et al. 2005, in Proc. 29th Cosmic Ray Conf. (Pune), 4, 435

Beloborodov, A. 2005, ApJ, 618, L13

Böttcher, M., \& Dermer, C. D. 1998, ApJ, 499, L131

Breiman, L. 2001, Machine Learning, 45, 5

Bretz, T., et al. (the MAGIC Collaboration). 2003, in Proc. 28th Int. Cosmic Ray Conf. (Tsukuba), 2943

. 2005, in Proc. 29th Int. Cosmic Ray Conf. (Pune), 4, 315

Burrows, D., et al. 2005, Science, 309, 1833

Chiang, J., \& Dermer, C. D. 1999, ApJ, 512, 699

Connaughton, V., et al. 1997, ApJ, 479, 859

Cortina, J., et al. (the MAGIC Collaboration). 2005, in Proc. 29th Int. Cosmic Ray Conf. (Pune), 5, 359

Cummings, J., et al. 2005, GCN Circ. 3910, http://gen.gsfc.nasa.gov/gen/gen3/ 3910.gen 3

de Jager, O. C., \& Stecker, F. W. 2002, ApJ, 566, 738

Derishev, E. V., Kocharovsky, V. V., \& Khocarovsky, Vl. V. 1999, ApJ, 521, 640 . 2001, A\&A, 372, 1071

Dermer, C. D., Chiang, J., \& Mitman, K. 2000, ApJ, 537, 785

Dingus, B. L. 1995, Ap\&SS, 231, 187

Durig, D. T. 2005, IAU Circ. 8521

Falcone, A., et al. 2005, GCN Circ. 3581, http://gcn.gsfc.nasa.gov/gen/gen3/ 3581.gcn 3

Fegan, D. J. 1997, J. Phys. G, 23, 1013

Fragile, P., et al. 2004, Astropart. Phys., 20, 591

Galante, N. 2006, Ph.D. thesis, Univ. Studi Siena, http://wwwmagic.mppmu .mpg.de/publications/theses/NGalante.pdf.gz

Galante, N., et al. 2005, GCN Circ. 3747, http://gcn.gsfc.nasa.gov/gen/gcn3/ 3747.gcn 3

Gaug, M. 2006, Ph.D. thesis, Univ. Autònoma Barcelona, http://magic.mppmu .mpg.de/publications/theses/MGaug.pdf

Gaug, M., et al. (the MAGIC Collaboration). 2005, in Proc. 29th Int. Cosmic Ray Conf. (Pune), 5, 375

Godet, O., et al. 2006, A\&A, 452, 819

Golenetskii, S., et al. 2005, GCN Circ. 3619, http://gcn.gsfc.nasa.gov/gcn/ $\mathrm{gcn} 3 / 3619$.gen 3

. 2006, GCN Circ. 4564, http://gen.gsfc.nasa.gov/gen/gen3/4564.gcn3

González, M. M., Dingus, B. L., Kaneko, Y., Preece, R. D., Dermer, C. D., \& Briggs, M. S. 2003, Nature, 424, 749

Götting, N., et al. 2003, GCN Circ. 1007, http://gcn.gsfc.nasa.gov/gcn/gcn3/ 1007.gen 3

Gotz, D., et al. 2005a, GCN Circ. 3323, http://gcn.gsfc.nasa.gov/gen/gen3/ 3323.gcn 3

. 2005b, GCN Circ. 3329, http://gcn.gsfc.nasa.gov/gcn/gcn3/3329.gcn3

Guetta, D., Piran, T., \& Waxman, E. 2005, ApJ, 619, 412

Guetta, D., et al. 2007, A\&A, 461, 95

Hengstebeck, T. 2006, Ph.D. thesis, Humboldt Univ. Berlin

Hillas, A. M. 1985, in Proc. 19th Int. Cosmic Ray Conf. (La Jolla), 3, 445

Horan, D., et al. 2007, ApJ, 655, 396
Hullinger, D., et al. 2005, GCN Circ. 3364, http://gcn.gsfc.nasa.gov/gen/gcn3/ 3364.gcn3

Hurkett, C., et al. 2005a, GCN Circ. 3360, http://gcn.gsfc.nasa.gov/gcn/gcn3/ 3360.gen 3

- 2005b, GCN Circ. 3379, http://gen.gsfc.nasa.gov/gen/gen3/3379.gen3 Hurley, K., et al. 1994, Nature, 372, 652

Jarvis, B., et al. (the STACEE Collaboration). 2005, in Proc. 29th Int. Cosmic Ray Conf. (Pune), 4, 455

Kawai, N., et al. 2005, GCN Circ. 3937, http://gcn.gsfc.nasa.gov/gcn/gen3/ 3937.gen3

Kobayashi, S., Zhang, B., Mészáros, P., \& Burrows, D. 2007, ApJ, 655, 391

Levan, A. J., et al. 2006, GCN Circ. 4841, http://gen.gsfc.nasa.gov/gen/gen3/ 4841.gcn3

Li, Z., Dai, G., \& Lu, T. 2002, A\&A, 396, 303

Malesani, D., et al. 2006, GCN Circ. 4561, http://gen.gsfc.nasa.gov/gen/gcn3/ 4561.gen 3

Mangano, V., et al. 2006, GCN Circ. 4565, http://gen.gsfc.nasa.gov/gen/gen3/ 4565.gcn3

Mannheim, K., Hartmann, D., \& Burkhardt, F. 1996, ApJ, 467, 532

Mereghetti, S., \& Mowlavi, N. 2005, GCN Circ. 4084, http://gcn.gsfc.nasa.gov/ gen $/ g c n 3 / 4084 . g c n 3$

Mészáros, P. 2006, Rep. Prog. Phys., 69, 2259

Mészáros, P., Rees, M., \& Papathanassoiu, H. 1994, ApJ, 432, 181

Mineo, T., et al 2005, GCN Circ. 3920, http://gcn.gsfc.nasa.gov/gcn $/ g c n 3 /$ 3920.gcn 3

Mirzoyan, R., et al. (the MAGIC Collaboration). 2005, in Proc. 29th Int. Cosmic Ray Conf. (Pune), 4, 23

Morris, D., et al. 2006, GCN Circ. 4682, http://gcn.gsfc.nasa.gov/gcn/gcn3/ 4682.gen 3

. 2007, ApJ, 654, 413

Nikishov, A. I. 1961, Zh. Eksp. Teor. Fiz., 41, 549 (English transl. in Soviet Phys.-JETP Lett., 14, 392 [1962])

Padilla, L., et al. 1998, A\&A, 337, 43

Palmer, D., et al. 2005, GCN Circ. 3597, http://gen.gsfc.nasa.gov/gen/gen3/ 3597.gcn 3

. 2006, GCN Circ. 4697, http://gcn.gsfc.nasa.gov/gcn/gcn3/4697.gcn3

Papathanassiou, H., \& Mészáros, P. 1996, ApJ, 471, L91

Pe'er, A., \& Waxman, E. 2004, ApJ, 613, 448

Pilla, R. P., \& Loeb, A. 1998, ApJ, 494, L167

Piran, T. 1999, Phys. Rep., 314, 575

Poirier, J., et al. 2003, Phys. Rev. D, 67, 042001

Preece, R. D., Briggs, M. S., Mallozzi, R. S., Pendleton, G. N., Paciesas, W. S., \& Band, D. L. 2000, ApJS, 126, 19

Razzaque, S., Mészáros, P., \& Zhang, B. 2004, ApJ, 613, 1072

Rolke, W., López, A., \& Conrad, J. 2005, Nucl. Instrum. Methods Phys. Res. A, 551, 493

Rossi, E., Beloborodov, A., \& Rees, M. J. 2006, MNRAS, 369, 1797

Sakamoto, T., et al. 2005, GCN Circ. 3938, http://gcn.gsfc.nasa.gov/gen/gen3/ 3938.gcn3

Saz Parkinson, P., et al. 2006a, in AIP Proc. 836, Gamma Ray Bursts in the Swift Era, ed. S. S. Holt, N. Gehrels, \& J. A. Nousek (New York: AIP), 624 . 2006b, GCN Circ. 5527, http://gcn.gsfc.nasa.gov/gcn/gcn3/5527.gcn3 2007, Nuovo Cimento, in press (astro-ph/0611457)

Scapin, V., et al. 2007, Nuovo Cimento, in press

Totani, T. 2000, ApJ, 536, L23

Wang, X., Dai, Z., \& Lu, T. 2001, ApJ, 556, 1010

Wang, X., Li, Z., \& Mészáros, P. 2006, ApJ, 641, L89

Waxman, E. 1995, Phys. Rev. Lett., 75, 386

Zhang, B., \& Mészáros, P. 2001, ApJ, 559, 110

Zhou, X., et al. 2003, in Proc. 28th Int. Cosmic Ray Conf. (Tsukuba), 2757 